Case report

\title{
Cardiac arrest from massive PE in nephrotic syndrome successfully treated with embolectomy and ECMO
}

\author{
Randi Connor-Schuler ${ }^{\mathrm{a}, *}$, Daniel Hrabec ${ }^{\mathrm{b}}$, Julio Pinto Corrales ${ }^{\mathrm{c}}$ \\ ${ }^{a}$ Henry Ford Hospital Department of Emergency Medicine, USA \\ ${ }^{\mathrm{b}}$ Henry Ford Hospital Department of Emergency Medicine/Internal Medicine, USA \\ ${ }^{\mathrm{c}}$ Henry Ford Hospital Department of Pulmonary Critical Care Medicine, USA
}

\section{A R T I C L E I N F O}

\section{Keywords:}

ECMO

Embolectomy

PE

Shock

Nephrotic syndrome

\begin{abstract}
A B S T R A C T
Pulmonary emboli (PE) are commonly encountered events with presentations ranging from benign incidental findings to obstructive shock. We present a case of a 20 year old male with nephrotic syndrome who suffered complete cardiovascular collapse with cardiac arrest in the setting of a massive PE, requiring open surgical embolectomy and ECMO support. We reviewed the literature on massive PE's focusing on the use of ECMO and success of the rarely performed open embolectomy for the treatment of obstructive shock from a massive PE.
\end{abstract}

\section{Case}

20 year old male with a history of nephrotic syndrome due to focal segmental glomerulosclerosis presented to the emergency department (ED) with complaints of abdominal discomfort and fatigue. The patient had a syncopal episode in the ED with head trauma and became tachycardic (138), hypotensive (98/50), tachypneic (28), and hypoxic requiring a non-rebreather mask. He was admitted to the MICU where he was found to be drowsy and mildly confused as well as cool to the touch with a swollen and edematous left lower extremity.

Work up was significant for a troponin level that increased from 0.06 to $2.87 \mathrm{ng} / \mathrm{mL}$, BNP was $150 \mathrm{pg} / \mathrm{mL}$, and EKG demonstrated a new right bundle branch block. Bedside ultrasound showed a large dilated RV with bowing septum, and STAT CT Pulmonary Embolism demonstrated a massive saddle pulmonary embolus with RV strain.

He was not considered a candidate for systemic IV tPA given he had a head injury with loss of consciousness following the syncopal episode that was associated with mental status changes and lack of intracranial imaging. Due to worsening hypoxia and decline in mental status, the patient was intubated; he subsequently became increasingly hypotensive. Hypotension was refractory to maximal vasopressor support with norepinephrine and epinephrine, and at that point cardiothoracic surgery was consulted who took the patient to the operating room for embolectomy.

The patient had a PEA arrest en route to the operating room for emergent open embolectomy. He received 30 minutes of open cardiac massage with surgical removal of a large clot burden (Fig. 1). Patient was then placed on VA ECMO due to RV failure and cor pulmonale. The patient was ultimately stabilized and able to be decannulated from VA ECMO 7 days later. He had a prolonged hospitalization requiring tracheostomy for ventilator dependent respiratory failure, acute kidney injury requiring renal replacement therapy, and encephalopathy. MRI did not show any significant abnormalities post cardiac arrest, and he gradually improved with complete neurologic function. Dialysis was discontinued, and tracheostomy was removed. On mycophenolate for nephrotic syndrome, he currently remains asymptomatic and able to perform and function independently.

\section{Discussion}

Pulmonary emboli (PE) are commonly encountered events that can be either acute or chronic in nature. Given the potential variation in size, location, and amount of clot burden, presentations can range from benign with an incidental finding of a PE to obstructive shock with complete cardiovascular collapse, such as in our case presentation.

There are many risk factors for PE, including prolonged immobilization or travel, pregnancy, estrogen use, or hypercoagulable disorders. Nephrotic syndrome is an uncommonly encountered but known associated risk factor for PE given the urinary losses of antithrombin and plasminogen with this disease process producing a hypercoagulable state [1]. Patients such as ours with nephrotic syndrome are thus at an increased risk for thromboembolic disease.

With the increasing frequency of diagnosed PE's, treatment modalities have also been evolving. Therapy can include anticoagulation

\footnotetext{
* Corresponding author.29900 Franklin Road \#112, Southfield, MI, 48034, USA.

E-mail addresses: RCONNOR1@hfhs.org (R. Connor-Schuler), DHRABEC1@hfhs.org (D. Hrabec), JPINTO1@hfhs.org (J.P. Corrales).
} 


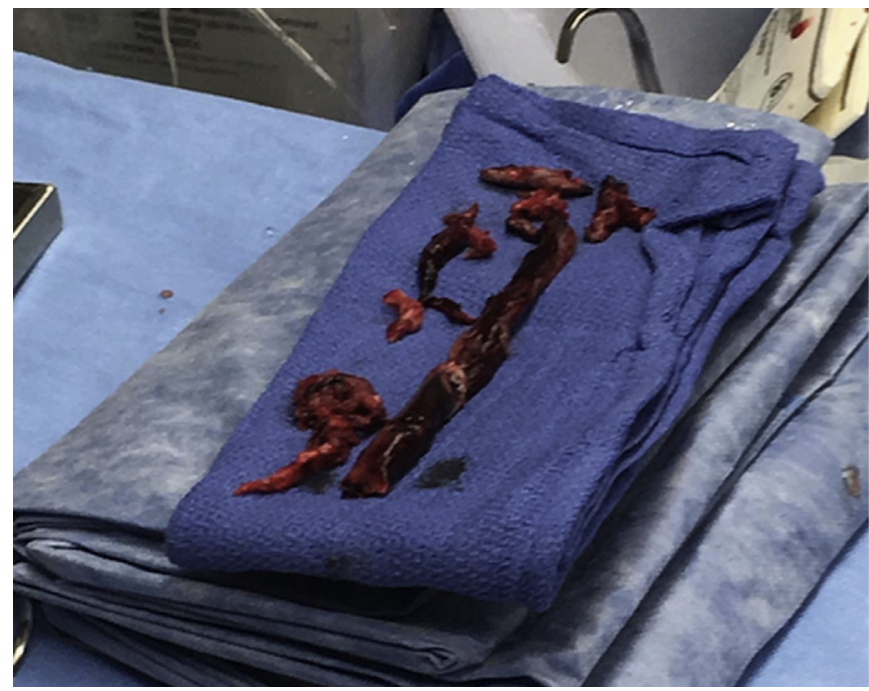

Fig. 1. Patient's clot burden removed in the OR during embolectomy.

with Coumadin or other novel anticoagulants for benign PE's, EKOS for submassive PE's, to systemic tPA or surgical embolectomy for massive PE's. Open embolectomies are rarely performed as patients are often either too ill to survive to the operating table or are well-enough for less invasive therapies such as catheter-directed lysis. In a review of 71 cases of massive PE's treated by open embolectomy by Gray et al., patients did well who underwent the surgery with $36 \%$ survival in patients who had arrested, $89 \%$ survival in patients who had not had a cardiac arrest and $70 \%$ of the survivors without significant morbidity [2]. In another case review by Dauphine and Omari, $73 \%$ of patients treated with embolectomy survived who had not arrested while only $9 \%$ of those suffering preoperative cardiac arrest survived [3]. Most of the patients who arrest suffer either neurologic insult or cardiogenic shock ultimately resulting in death. Our patient's case provides further evidence for potential positive outcomes with a surgical embolectomy, including patients following a cardiac arrest.

Additionally, following cardiac arrest and embolectomy our patient had complete RV failure, for which he was placed on VA ECMO support. Research and reports are scarce, however ECMO has been performed in cases of massive PE as a temporizing measure to embolectomy [4,5] as well as for ongoing RV failure following embolectomy [6].

Massive PE's causing obstructive shock are uncommonly encountered and are often fatal due to complete cardiopulmonary collapse. Surgical embolectomies are rarely performed, however are associated with positive outcomes as demonstrated by literature review. Patients requiring such advanced therapies do not typically survive to time of operation, however ECMO has been observed as a method of support to allow the patient to make it to the operating table as well as survive the RV failure that can occur postoperatively, as observed in our case.

\section{Author Contributions}

Dr. Connor-Schuler and Dr. Hrabec contributed equally with regards to preparation of this case study.

\section{Conflicts of interest}

No financial disclosures or conflicts of interest were present.

\section{Appendix A. Supplementary data}

Supplementary data related to this article can be found at http://dx. doi.org/10.1016/j.rmcr.2018.05.021.

\section{References}

[1] R.H. Kauffmann, J.J. Veltkamp, N.H. Tilburg, et al., Acquired antithrombin III deficiency and thrombosis in the nephrotic syndrome, Am. J. Med. 65 (4) (October 1978) 607-613.

[2] H.H. Gray, J.M. Morgan, M. Paneth, et al., Pulmonary embolectomy for acute massive pulmonary embolism: an analysis of 71 cases, Br. Heart J. 60 (1988) 196-200.

[3] C. Dauphine, B. Omari, Pulmonary embolectomy for acute massive pulmonary embolism, Ann. Thorac. Surg. 79 (4) (April 2005) 1240-1244.

[4] P.C. Hseih, S.S. Wang, W.J. Ko, et al., Successful resuscitation of acute massive pulmonary embolism with extracorporeal membrane oxygenation and open embolectomy, Ann. Thorac. Surg. 72 (1) (July 2001) 266-267.

[5] G. Lebreton, N. Bouabdallaoui, L. Gauduchon, et al., Successful use of ECMO as a bridge to surgical embolectomy in life-threatening pulmonary embolism, Am. J. Emerg. Med. 33 (9) (2015) 1332.e3-1332.e4.

[6] J. Belohlavek, V. Rohn, P. Jansa, et al., Veno-arterial ECMO in severe acute right ventricular failure with pulmonary obstructive hemodynamic pattern, $J$ Invasive Cardiol. 22 (8) (August 2010) 365-369. 